\title{
Estudo e análise estatística no mercado de ações brasileiro
}

\author{
Jonis Jecks Nervis \\ Mestre em Engenharia de Produção - Faculdade de Engenharia - Unesp Bauru \\ Departamento de Matemática, Universidade Estadual do Norte do Paraná \\ (UENP) \\ Endereço: Rua Conselheiro Nabuco, 74 Centro \\ CEP: 18950-000 Ipaussu - SP Brasil \\ e-mail: jonisjn@uenp.edu.br
}

\author{
Antonio Fernando Crepaldi \\ Doutor em Física, Instituto de Física Teórica, UNESP \\ Departamento de Engenharia de Produção, Faculdade de Engenharia de \\ Bauru, UNESP \\ Endereço: Av. Eng. Luiz Edmundo C. Coube 14-01 Vargem Limpa \\ CEP: 17033-360 Bauru - SP Brasil \\ e-mail: crepaldi@feb.unesp.br
}

\begin{abstract}
Resumo
O objetivo dessa pesquisa é verificar e analisar a existência no Brasil dos principais fatos estilizados observados em séries temporais financeiras: agrupamento de volatilidade, distribuições de probabilidades com caudas gordas, presença de memória de longo alcance na série temporal dos retornos absolutos, ausência de autocorrelação linear dos retornos, assimetria ganho/perda, gaussianidade agregativa, decaimento lento da autocorrelação dos retornos absolutos, correlação volume/volatilidade e efeito de alavancagem. Para isso, foram utilizadas e analisadas as cotações intraday de ações de dez companhias negociadas na Bolsa de Valores, Mercadorias e Futuros que correspondem juntas a uma participação de 52,1\%, para a data de 01/09/2009, no Índice Bovespa. A partir da análise dos dados foi possível verificar a existência dos fatos estilizados e que eles apresentam um comportamento compatível com os dos mercados internacionais.
\end{abstract}

Palavras-chave: Fatos estilizados em mercados financeiros; Dados financeiros de alta frequência; Séries temporais do mercado de ações brasileiro.

\begin{abstract}
The objective of this paper is to verify and analyze the existence in Brazil of stylized facts observed in financial time series: volatility clustering, probability distributions with fat tails, the presence of long run memory in absolute return time series, absence of linear return autocorrelation, gain/loss asymmetry, aggregative gaussianity, slow absolute return autocorrelation decay, trading volume/volatility correlation and leverage effect. We analyzed intraday prices for 10 stocks traded at the BM\&FBovespa, responsible for $52.1 \%$ of the Ibovespa portfolio on Sept. 01, 2009. The data analysis confirms the stylized facts, whose behavior is consistent with what is observed in international markets.
\end{abstract}

Keywords: Stylized facts in financial markets; High frequency data; Brazilian stock market time series. 


\section{Introdução}

O conhecimento da natureza estatística das séries temporais é importante para o mercado de ativos, tanto no que concerne ao cálculo de risco, bem como ao mecanismo de formação de preços de derivativos e outros instrumentos financeiros. Um dos principais interesses na área de finanças está no desafio científico de compreender a dinâmica das grandes flutuações em sistemas complexos com um grande número de elementos interagentes (GOPIKRISHNAN et al., 1999).

Dois aspectos importantes que possibilitaram um aumento do número de pesquisas em séries financeiras foram a crescente disponibilidade de dados de alta frequência (bases de dados com frequência de segundos) sobre os preços praticados nos mais diversos tipos de mercados e a utilização de métodos computacionais (CONT, 2001; MANTEGNA e STANLEY, 2000; BOUCHAUD e POTTERS, 2003). A análise desses dados permitiu a observação de um conjunto de características estatísticas que são comuns aos vários tipos de instrumentos, mercados, e em diferentes períodos. Essas propriedades ou características são chamadas de fatos estilizados, segundo Cont (2001), e são condizentes com a possibilidade da existência de resultados "universais" (GOPIKRISHNAN et al., 1999). Elas não estão associadas a nenhuma técnica paramétrica aplicada aos dados e, portanto, fornecem somente informações qualitativas sobre as séries utilizadas. Contudo, os fatos estilizados são tão restritivos que se torna difícil gerar séries estocásticas que possuam tais propriedades (CONT, 2001). Apesar de alguns pesquisadores, no passado, tomarem a abordagem de caos para tratar as séries financeiras, elas são mais bem modeladas por processos estocásticos (MANTEGNA e STANLEY, 2000). Os físicos buscam caracterizar esses processos investigando sua correlação temporal e estudando o comportamento assintótico de sua função de distribuição de probabilidade (MANTEGNA e STANLEY, 2000).

Uma crítica feita às análises financeiras que se baseiam na causalidade dos movimentos do mercado financeiro, a partir de fatos econômicos e políticos, é que diferentes ativos não são necessariamente influenciados pelos mesmos conjuntos de eventos informacionais (CONT, 2001). Sendo assim, as séries de preços dos diferentes ativos exibiriam propriedades diversas, ou seja, caberia o seguinte tipo de pergunta: por que as propriedades estatísticas dos preços futuros de commodities seriam similares às do comportamento de preços de ações de empresas de informática ou da taxa de câmbio? No entanto, as pesquisas com dados empíricos apontam a existência dos fatos estilizados permeando os diversos tipos de mercados financeiros, conforme afirmam Cont (2001), Mantegna e Stanley (2000), e Bouchaud e Potters (2003). Dentre eles, podem-se citar como os mais importantes: ausência de autocorrelação linear dos retornos, caudas gordas, assimetria ganho/perda, gaussianidade agregativa, agrupamento de volatilidade, decaimento lento da autocorrelação dos retornos absolutos, correlação volume/volatilidade e efeito de alavancagem. 
Neste trabalho realizou-se um estudo qualitativo empírico de verificação de propriedades e comportamentos do mercado financeiro brasileiro, caracterizando, portanto, um problema sem a construção de hipóteses e, consequentemente, de verificação e análise do comportamento de uma variável escolhida.

\section{Fatos estilizados do retorno}

Entre as medidas de variação de preços, aquela que é mais largamente utilizada em estudos de séries temporais financeiras é o retorno $r(t)$. Portanto, para iniciar uma análise das propriedades estatísticas de uma série financeira, o primeiro trabalho a ser feito é transformar a série de preços em uma série de retornos.

Define-se o conceito de variação relativa de preços ou retorno por:

$$
r(t, \Delta t)=\ln P(t+\Delta t)-\ln P(t)
$$

onde $P$ é o preço, $t$ é o tempo e $\Delta t$ o intervalo de tempo entre dados sucessivos da série.

A seguir, faz-se uma caracterização de um conjunto de fatos estilizados estatísticos relacionados ao retorno que são comuns a um vasto conjunto de ativos financeiros.

\subsection{Ausência de autocorrelação linear dos retornos}

A autocorrelação é uma medida que informa o quanto o valor de uma realização de uma variável aleatória é capaz de influenciar seus valores vizinhos; por exemplo, o quanto a existência de um valor mais alto condiciona valores também altos de seus vizinhos.

A função de autocorrelação é dada por:

$$
C(\tau)=\operatorname{corr}(r(t, \Delta t), r(t+\tau, \Delta t))
$$

onde $\mathrm{C}$ significa a autocorrelação, $\mathrm{r}$ o retorno, $\tau$ o incremento temporal, t o tempo e corr a correlação dada por:

$$
\operatorname{corr}(X, Y)=\frac{E[X Y]-E[X] E[Y]}{\sigma_{X} \sigma_{Y}}
$$

em que $E[$ ] é a esperança matemática e $\sigma$ o desvio padrão.

A ausência de autocorrelação linear nos incrementos de preços para retornos de mercado tem sido amplamente documentada, segundo Cont (2001), Mantegna e Stanley (2000) e Fama (1970).

Para os retornos de mercado, a função de autocorrelação apresenta uma tendência rápida para valores próximos de zero (GOPIKRISHNAN et al., 1999, MANTEGNA e STANLEY, 2000). Ou seja, verificam-se valores significati- 
vos de autocorrelação apenas para escalas de tempo menores que 20 minutos (CONT, 2001).

\subsection{Caudas gordas}

Cauda gorda é uma propriedade de algumas distribuições de probabilidade, exibindo curtose extremamente grande comparativamente à distribuição normal. O excesso de curtose $(k)$ é uma medida do achatamento de uma distribuição e é representada por:

$$
k=\frac{E[r(t, \Delta t)-E[r(t, \Delta t)]]^{4}}{\sigma_{r}^{4}(t)}-3
$$

Como a curtose baseada no quarto momento centrado na média apresenta valor igual a três para a distribuição normal, ao se subtrair esse número do valor encontrado se obtém valor positivo para uma distribuição leptocúrtica, valor negativo para uma distribuição platicúrtica, e para uma distribuição normal o valor encontrado é nulo.

Assim definido, o excesso de curtose para uma distribuição gaussiana é nulo, $k=0$, e para distribuições que apresentam caudas gordas, $k>0$.

Para algumas classes de eventos a probabilidade de ocorrência de realizações extremas é relativamente alta. Mantegna e Stanley (2000) afirmam que, quanto maior a frequência dos dados maior é o grau de leptocurtose observado. Eles também evidenciaram, para ações da Xerox negociadas na Bolsa de Valores de New York, a leptocurtose naturalmente observada nos dados empíricos e as diferenças observadas entre a distribuição empírica do retorno em comparação com a distribuição de probabilidade gaussiana.

\subsection{Assimetria ganho/perda}

A medida de assimetria da distribuição de probabilidades de uma variável aleatória real, segundo Spiegel (1985), é:

$$
\text { Assimetria }=\frac{E[r(t, \Delta t)-E[r(t, \Delta t)]]^{3}}{\sigma_{r}^{3}(t)}
$$

A assimetria de uma distribuição normal é igual a zero. Valores negativos para a assimetria indicam dados que são enviesados à esquerda, e valores positivos para a assimetria indicam dados que são distorcidos à direita.

\subsection{Gaussianidade agregativa}

A gaussianidade agregativa, além de descrever uma série de fenômenos físicos e financeiros, possui grande uso na estatística inferencial, e é inteiramente 
descrita por seus parâmetros de média $(\mu)$ e desvio padrão $(\sigma)$. As distribuições gaussianas são definidas a partir de uma função densidade de probabilidade que se escreve da seguinte forma:

$$
P(x)=\frac{1}{\sqrt{2 \pi \sigma}} e^{-\frac{(x-\mu)^{2}}{2 \sigma^{2}}}
$$

Onde x é a variável estocástica em questão, $\mu=E[r(t, \Delta t)]$ é a média da distribuição, e $\sigma^{2}=E(r(t, \Delta t))^{2}-E^{2}[r(t, \Delta t)]$ é a variância.

A convergência para uma distribuição gaussiana, que os dados de mercado apresentam quando são tomados de intervalos cada vez maiores de tempo, é chamada de gaussianidade agregativa (CONT, 2001).

\subsection{Decaimento lento da autocorrelação dos retornos absolutos}

A função de autocorrelação temporal $C(\tau)$ é definida como na equação (2).

Funções não lineares, como o valor absoluto ou o quadrado dos retornos, apresentam autocorrelação positiva, segundo Cont (2001). A amplitude dos retornos (valor absoluto do retorno), por exemplo, mostra correlação que persiste por vários meses e é caracterizada por um decaimento assintótico com lei de potência (GOPIKRISHNAN et al., 1999). Esse comportamento é apresentado por Gopikrishnan et al. (1999), que demonstra que a função log-log de autocorrelação dos retornos absolutos do índice S\&P 500, em uma escala temporal com $\Delta t=1 \mathrm{~min}$ apresenta decaimento lento seguindo uma lei de potência.

\section{Fatos estilizados da volatilidade}

A flutuação média do preço de mercado em dado período corresponde à volatilidade (CIZEAU, 1997), sendo de grande importância na determinação do risco. Além disso, Mantegna e Stanley (2000), Bouchaud e Potters (2003) e Cizeau (1997) lembram que a volatilidade é parâmetro chave nos modelos de apreçamento de opções.

Apesar do grande interesse prático, a volatilidade não é diretamente observável (BOUCHAUD e POTTERS, 2003). O que se faz é estimá-la por alguma forma de aproximação. É possível descrevê-la através de uma função log-normal (CIZEAU, 1997).

Como no decorrer do dia o mercado mostra um comportamento em forma de "U", devido à alta atividade no início da manhã e no fim da tarde e à baixa atividade durante a tarde, é necessário remover a correlação artificial resultante desse padrão de volatilidade intraday.

Para tanto é necessário normalizar a função (CIZEAU, 1997):

$$
g(t)=\frac{G(t)}{A(t)}
$$


onde $A(t)$ é média dos valores de $|G(t)|$, tomados ao mesmo tempo do dia e calculada a média sobre todos os dias do conjunto de dados. Já $G(t)$ é o logaritmo dos incrementos:

$$
G(t)=\ln Z(t+\Delta t)-\ln Z(t)
$$

em que $Z(t)$ denota o índice de tempo $t$ e $\Delta t$ é o intervalo de tempo, ou seja, $G(t)$ é a mudança relativa de preço $\frac{\Delta Z}{Z}$ quando $\Delta t \rightarrow 0$. Nesse estudo, $\Delta(t)=20 \mathrm{~min}$.

É obtida a volatilidade em um dado momento através da média de $G(t)$ sobre uma janela de tempo $T=n \Delta t$ com algum valor inteiro para $n$.

$$
v_{T}(t)=\frac{1}{n} \sum_{t^{\prime}=t}^{t+n-1}\left|G\left(t^{\prime}\right)\right|
$$

\subsection{Agrupamento de volatilidade}

A volatilidade é uma medida da intensidade e da frequência das oscilações nas cotações de um ativo financeiro, o qual pode ser uma ação, um título de renda fixa, a quota de um fundo de investimento, ou ainda, o valor de um índice de bolsa de valores, considerado um determinado período. Esta medida é um dos parâmetros mais frequentemente utilizados como forma de mensurar o risco de um determinado ativo (MORGAN, 1996).

A volatilidade é a quantidade e intensidade de flutuações e oscilações que ocorrem com uma série de retornos. Agrupamentos de volatilidade são séries temporais caracterizadas por grandes e intermitentes flutuações. Cont (2001) apresenta esse comportamento para ações da BMW na bolsa de valores de Frankfurt no período de 1992-1998.

\subsection{Correlação entre volume e volatilidade}

Plerou et al. (2000) e Silva et al. (2010) mostram que a correlação retorno(r)volume(v) tem o mesmo comportamento que a alavancagem $(\operatorname{Corr}(r-v)<0)$, significando que quedas de retornos estão associadas a um aumento na atividade de negociação.

Esse comportamento está em conformidade com o apresentado por Gopikrishnan et al. (1999) e Cont (2001) em que, na análise das propriedades estatísticas do número de ações negociadas para uma dada ação estudada em um intervalo de tempo fixo, tem-se que o volume de negócios está correlacionado com todas as medidas de volatilidade.

\subsection{Efeito de alavancagem}

$\mathrm{O}$ efeito de alavancagem explica a rentabilidade de uma empresa em termos do retorno sobre o capital empregado e do custo da dívida. Segundo Black 
(1976), a maioria das medidas de volatilidade de um ativo é negativamente correlacionada com os seus preços passados. Desta forma, em geral a volatilidade é maior em momentos de baixa de seus papéis, possivelmente devido ao aumento subsequente da alavancagem da companhia (endividamento em relação ao valor do ativo total) nestes períodos.

Essa medida de dependência não linear é definida por Cont (2001) da seguinte maneira:

$$
L(\tau)=\operatorname{corr}\left(r(t+\tau, \Delta t)^{2}, r(t, \Delta t)\right)
$$

A correlação dos retornos com seus quadrados tem valor negativo e decai a zero com o aumento de $\tau$. Isso sugere que retornos negativos levam a um aumento da volatilidade e retornos positivos a diminuem.

Segundo Bouchaud e Potters (2003) a correlação entre retorno e volatilidade é de curto alcance e o seu decaimento temporal está em torno de 10 dias, para índices de ações, e de aproximadamente 50 dias para ações individuais. A amplitude da correlação também é diferente nos dois casos, tendendo a ser maior para índices de ações do que para ações individuais.

Ainda em Bouchaud e Potters (2003) encontra-se o ajuste da função correlação por uma exponencial:

$$
L(l)=-A e^{l / T}
$$

onde $A$ é a amplitude, $l$ o incremento temporal e $T$ o período. Verificou-se que o efeito de alavancagem para índices tem maior amplitude $(A=18 ; T=9,3$ dias), e tende a zero mais rapidamente com $l$, comparativamente ao comportamento de ações individuais $(A=1,8 ; T=69$ dias $)$.

\section{Dados e metodologia}

No Brasil, a negociação de compra e venda de ações é realizada na Bolsa de Valores, Mercadorias e Futuros (BM\&FBOVESPA), na qual se pode comprar e vender ações de companhias abertas. A BM\&FBOVESPA é uma associação civil sem fins lucrativos, com autonomia administrativa, financeira e patrimonial.

O índice composto pelas ações mais representativas negociadas na bolsa de valores brasileira é chamado de IBOVESPA. Implantado em 02/01/1968, é hoje o mais importante termômetro do desempenho médio das cotações do mercado de ações brasileiro. Ele retrata o comportamento dos principais papéis negociados na BM\&FBOVESPA. Para escolher esses papéis, a BM\&FBOVESPA considera o número de negócios e o volume movimentado nos 12 meses imediatamente anteriores.

O índice é composto por uma carteira teórica de ações que representam cerca de $80 \%$ do volume total negociado. Há atualmente 428 companhias abertas listadas na Bolsa, mas nem todas têm suas ações negociadas diariamente. A 
composição do IBOVESPA é reavaliada a cada quatro meses, nos meses de janeiro, maio e setembro. Isso ocorre devido ao objetivo de se manter um índice representativo do mercado.

Construímos esta pesquisa utilizando cotações intraday de ações de 10 companhias negociadas na Bolsa de Valores, Mercadorias e Futuros (BM\&FBOVESPA). Os dados referentes a essas empresas são dados primários, obtidos diretamente da BM\&FBOVESPA. Essas companhias correspondem a uma participação, divulgada para a abertura dos negócios do dia 01/09/2010, de $52,1 \%$ na carteira do Ibovespa.

O período de análise das cotações vai de 01/07/2006 até 30/11/2008, ou seja, 29 meses. A escolha das empresas foi de acordo com o critério de maior liquidez que elas apresentam perante o Ibovespa na data de 01/09/2010.

Todos os resultados foram obtidos a partir dos retornos das ações escolhidas, considerando sua importância na formação do Ibovespa em 01/09/2010. Indexamos o tempo ao número de transações que ocorrem; assim, privilegiamos a ocorrência sucessiva do número de transações, ou seja, utilizamos o método tick-by-tick.

Foram desenvolvidos dois programas: o primeiro com a função de buscar e coletar as informações relativas ao tempo em que a operação ocorreu e o valor correspondente dessa operação. O segundo programa teve como objetivo coletar amostras em intervalos de tempo iguais. O intervalo de tempo definido foi de vinte minutos (1200 segundos) para coleta ocorrendo da seguinte forma: quando o programa começa a rotina de programação ele busca o primeiro dado da amostra; a seguir, ele evolui no tempo vinte minutos e coleta outra informação. Não existe o risco de não se obter um dado, pois o número de operações foi indexado ao tempo. É nos dados obtidos pelo segundo programa que se baseia o estudo. Na Tabela 1 estão listadas as ações escolhidas e a sua participação no Ibovespa em 01/09/2010. Na primeira coluna têm-se os códigos que identificam as empresas nas transações de compra e venda. Na segunda coluna é apresentado o nome da empresa.

Os tipos de ações ocupam a terceira coluna, sendo ON ações ordinárias, que dão direito a voto em assembleias de acionistas. Já as ações PN (ações preferenciais) não dão direito a voto ou restringem esse direito de alguma forma. Investidores possuidores de ações PN tem preferência no recebimento de dividendos e/ou outros proventos distribuídos pela empresa. Existe a ação PNA que indica uma ação PN preferencial nominativa de classe A. A classe A pode indicar que seja uma ação com dividendo mínimo, e classe B com dividendo fixo. Outras letras ou números podem acompanhar o tipo de ação, pois as empresas podem emitir diferentes classes estabelecendo, em seu estatuto, valores diferenciados de dividendos ou proventos especiais para cada classe de ação (BM\&FBOVESPA, 2010).

\section{Resultados}

A seguir são apresentados os resultados obtidos no trabalho. Iniciamos pela autocorrelação, que é uma medida que informa o quão intensamente o valor de 
Tabela 1.- Ações consideradas no estudo.

\begin{tabular}{cccc}
\hline Código & Nome da Empresa & Tipo & Partic. $\%$ \\
\hline BBAS3 & Banco do Brasil & ON NM & 2,124 \\
\hline BBDC4 & Bradesco & PN N1 & 3,864 \\
\hline CSNA3 & Sid. Nacional & ON & 3,023 \\
\hline GGBR4 & Geran & PN N1 & 3,647 \\
\hline ITSA4 & Itaú AS & PN EDJ N1 & 2,455 \\
\hline PETR3 & Petrobras & ON & 3,336 \\
\hline PETR4 & Petrobras & PN & 15,012 \\
\hline USIM5 & Usiminas & PNA N1 & 3,100 \\
\hline VALE3 & Vale & ON N1 & 3,468 \\
\hline VALE5 & Vale & PNA NI & 12,076 \\
\hline & TOTAL & & $\mathbf{5 2 , 1 0 5}$
\end{tabular}

uma realização de uma variável aleatória é capaz de influenciar seus valores vizinhos. Ela permite que se analise o grau de irregularidade de um sinal, apresentando para retornos de mercado uma tendência rápida para valores próximos de zero, o que implica ausência de autocorrelação. Na Figura 1 é fácil a verificação desse comportamento, pois todos os valores observados, utilizando um intervalo de tempo $\Delta t=20 \mathrm{~min}$, flutuam na vizinhança da reta que representa o zero.

O excesso de curtose para uma distribuição gaussiana é nulo, e para distribuições que apresentam caudas gordas ele assume valores maiores que zero $(k$ $>0)$. Na Tabela 2 têm-se os valores para o excesso de curtose. Como é possível verificar, para todas as empresas o valor da curtose é maior que zero, caracterizando uma função densidade de probabilidade leptocúrtica, ou seja, que a distribuição apresenta caudas pesadas.

Tabela 2.- Excesso de curtose (k) para cinco ações da amostra.

\begin{tabular}{cccc}
\hline Código & Valor de $\mathbf{K}$ & Código & Valor de $\mathbf{K}$ \\
\hline BBAS3 & 53,4257 & PETR3 & 35,4884 \\
\hline BBDC4 & 57,1575 & PETR4 & 52,0813 \\
\hline CSNA3 & 57,3345 & USIM5 & 50,0814 \\
\hline GGBR4 & 38,8004 & VALE3 & 51,2916 \\
\hline ITSA4 & 78,3183 & VALE5 & 49,1413 \\
\hline
\end{tabular}


Figura 1.- Autocorrelações dos retornos das três ações mais significativas da amostra.

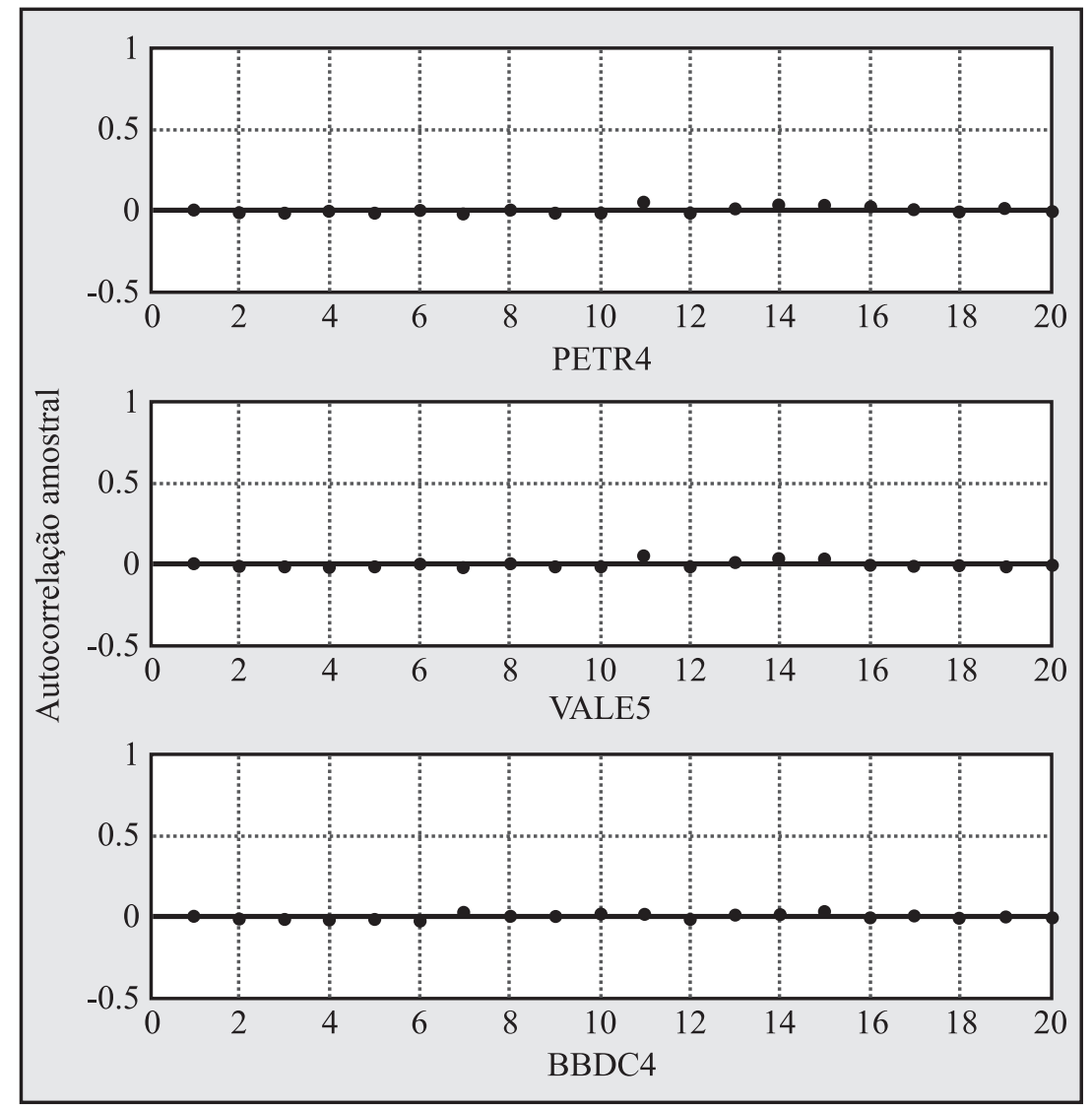

A Figura 2 apresenta o histograma dos retornos das ações VALE3 com a distribuição gaussiana (linha contínua) sobreposta, permitindo visualisar que os dados divergem de um comportamento gaussiano. Visualiza-se um pico acentuado extrapolando os limites da distribuição gaussiana.

Ações de empresas distintas apresentam variações absolutas de preços muito discrepantes em um período; portanto, o retorno apresenta-se com grande variabilidade nesse período. Na Figura 3 apresentamos os retornos das oito ações com maior percentual na carteira do Ibovespa. Percebe-se no final do período observado uma acentuada variabilidade do retorno. Esse comportamento coincide com a crise financeira internacional de 2008, desencadeada pela falência do banco de investimento estadunidense Lehman Brothers, no processo que ficou conhecido como crise do subprime. 
Figura 2.- Distribuição gaussiana ajustada ao histograma dos retornos de VALE3.

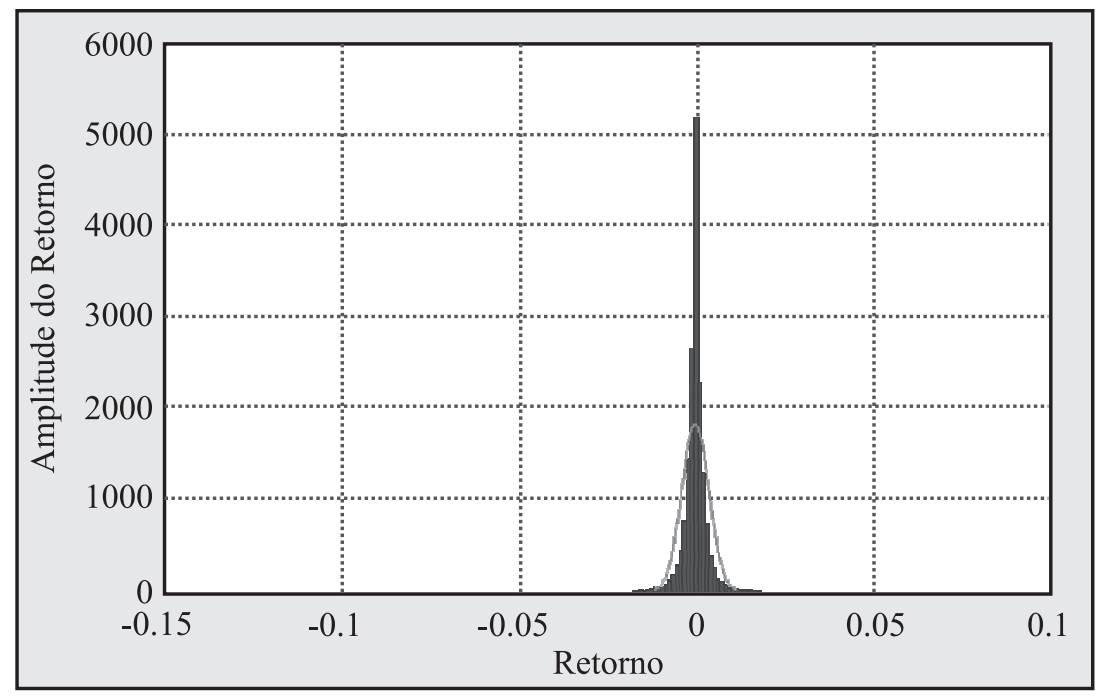

O lento decaimento da função de autocorrelação dos retornos absolutos é observável no gráfico da Figura 4, que apresenta os dados em escala normal. Estão representadas as três ações mais importantes da amostra, com escala temporal de vinte minutos.

Quando uma distribuição é normal, a assimetria dessa distribuição assume valor igual a zero. Porém, se o valor encontrado para a assimetria é negativo, isso significa que se tem dados enviesados à esquerda, enquanto valores positivos para a assimetria indicam dados distorcidos à direita. Na Tabela 3 são fornecidos os valores obtidos para a assimetria dos retornos das ações que compõem a amostra. É possível verificar assimetria à esquerda para os dados analisados.

A gaussianidade agregativa corresponde a uma aproximação para a distribuição gaussiana quando o intervalo de tempo entre as coletas de dados torna-se maior. A Figura 5 apresenta três gráficos das ações PETR4, começando com amostras coletadas com intervalo de vinte minutos, seguido do gráfico para amostras coletadas com intervalo de três horas, e finalizando com o gráfico para amostras coletadas com intervalo de trinta e seis horas. Foi realizado o teste KS para verificar o comportamento dessas séries em termos de normalidade. Supôs-se, para a hipótese nula $\left(\mathrm{H}_{0}=0\right)$, que os dados se assemelham à curva gaussiana, e para $\mathrm{H}_{1}=1$ a não semelhança. Para o primeiro gráfico (vinte minutos) e o segundo gráfico (três horas), obteve-se valor 1 no teste KS. Para o terceiro gráfico (trinta e seis horas), o teste KS forneceu valor 0; ou seja, com o aumento do intervalo de coleta de dados a distribuição tende a uma curva gaussiana. 
Figura 3.- Retornos diários das ações da amostra no período analisado.
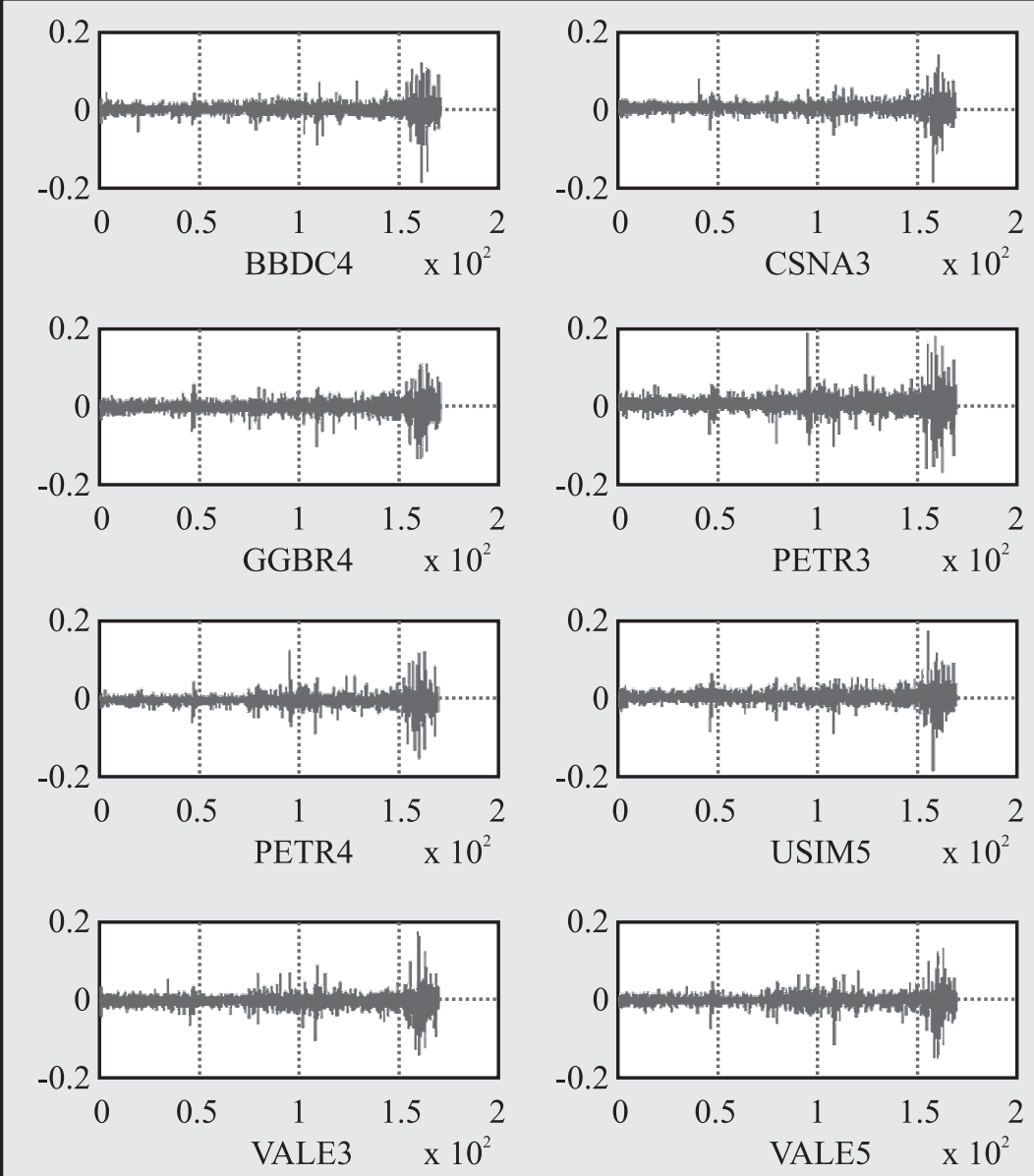

Realizamos o estudo da normalidade das distribuições por meio do teste de normalidade de Kolmogorov-Smirnov (Teste KS). Adotou-se para todos os testes o nível de significância $\alpha$ de cinco por cento de probabilidade para a rejeição da hipótese nula $\left(\mathrm{H}_{0}=0\right)$, que configura a normalidade dos dados, sendo que $\mathrm{H}_{0}=0$ significa não rejeitar a hipótese nula no nível de significância $\alpha$, e $\mathrm{H}_{1}=$ 1 rejeitar a hipótese nula no nível de significância $\alpha$.

O resultado do teste KS acima descrito, realizado com todas as ações que compõem a amostra, mostrou que não houve significância para as variáveis estudadas com o recurso apresentado nas duas hipóteses; ou seja, obteve-se resultado favorável a $\mathrm{H}_{1}=1 \mathrm{em}$ todos os casos. Com isso concluiu-se pela não normalidade dos dados estudados. 
Figura 4.- Função de autocorrelação dos retornos absolutos das ações mais importantes da amostra, com escala temporal de $\Delta t=20 \mathrm{~min}$.

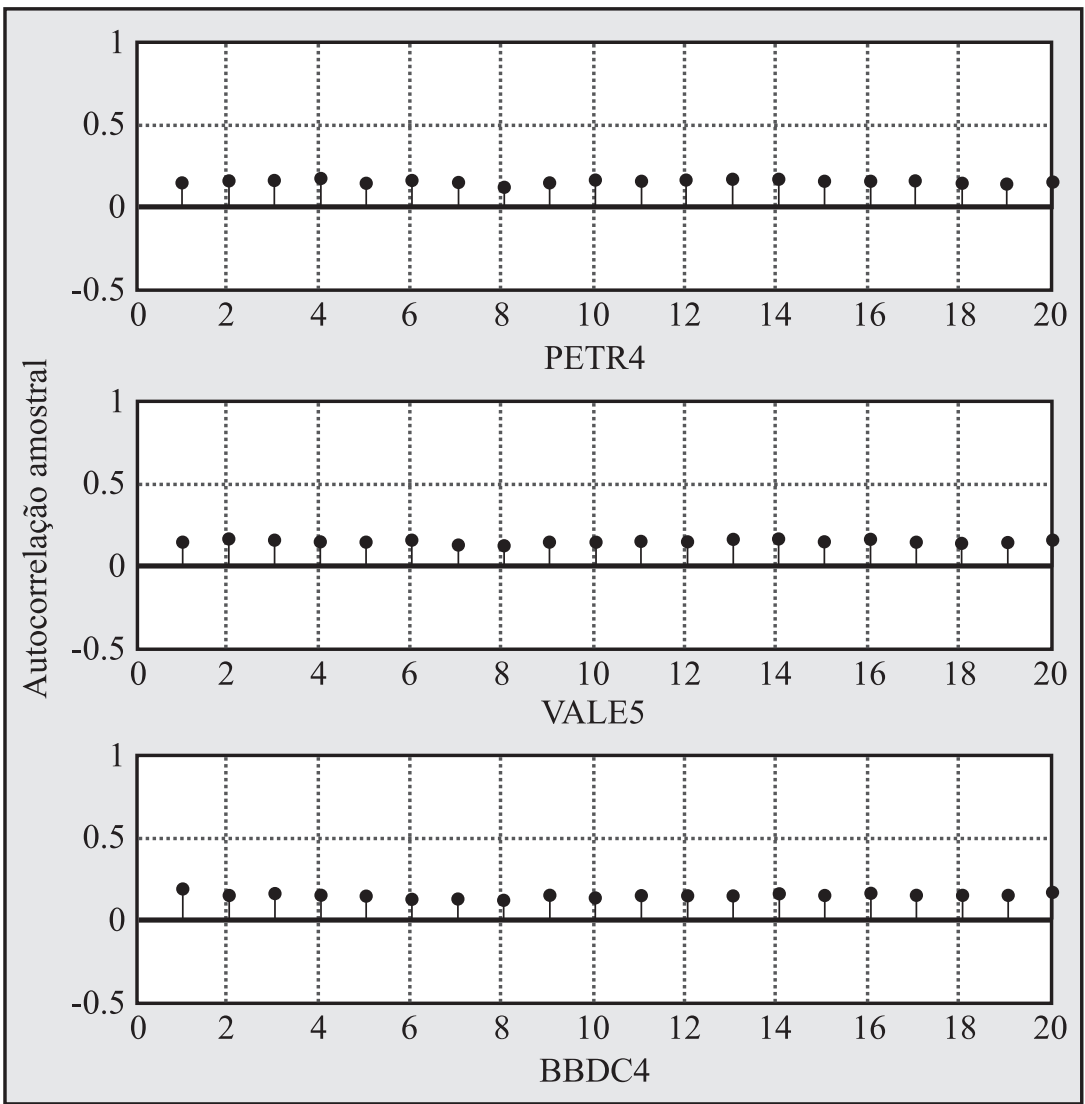

Tabela 3.- Assimetria de retornos das ações da amostra.

\begin{tabular}{cc}
\hline Código & Assimetria \\
\hline BBAS3 & $-1,0618$ \\
BBDC3 & $-0,6332$ \\
CSNA3 & $-0,4932$ \\
GGBR4 & $-0,7673$ \\
ITSA4 & $-0,1337$ \\
PETR3 & $-0,1001$ \\
PETR4 & $-0,0677$ \\
USIM5 & $-0,4634$ \\
VALE3 & $-0,3540$ \\
VALE5 & $-0,6841$ \\
\hline
\end{tabular}


Figura 5.- Histogramas de PETR4 para verificar a existência de gaussianidade agregativa.

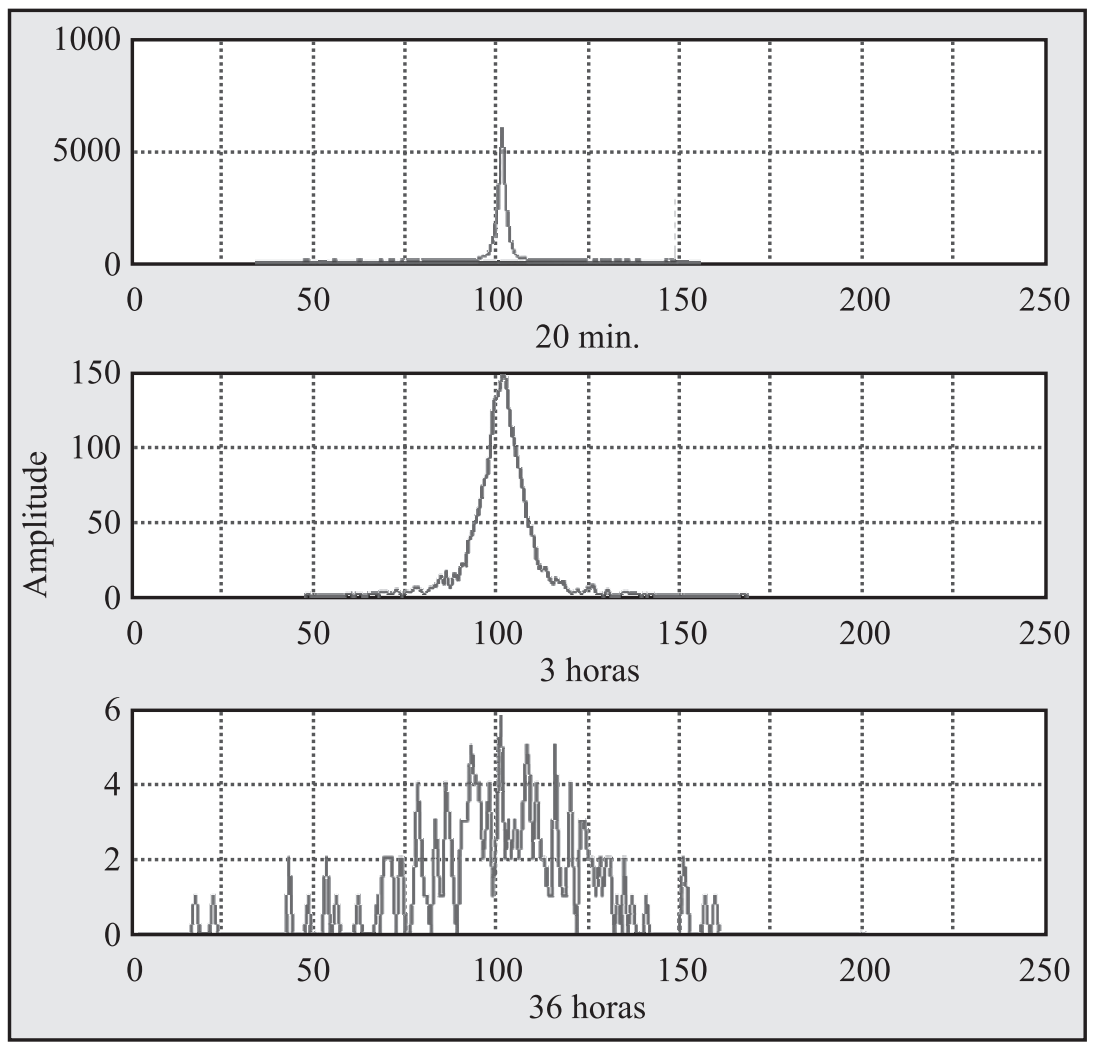

A volatilidade não é diretamente observável, segundo Bouchaud e Potters (2003). O que se faz é estimá-la por alguma forma de aproximação ou ainda descrevê-la através de uma função log-normal, conforme Cizeau (1997).

Como no decorrer do dia o mercado mostra um comportamento em forma de "U" devido à alta atividade no inicio da manhã e no fim da tarde e à baixa atividade durante a tarde, é necessário remover a correlação artificial resultante desse padrão de volatilidade intraday.

A Figura 6 apresenta o melhor ajuste log-normal com a distribuição de volatilidade das ações CSNA3, calculado numa janela de tempo de $300 \mathrm{~min}$.

Efetuou-se um teste Qui-Quadrado para a aceitação do modelo log-normal em relação à volatilidade em todas as ações que compõem a amostra. Este teste estatístico consiste em calcular a soma dos desvios quadráticos do modelo hipotético em relação aos dados empíricos, obtendo-se o valor de $X_{0}^{2}$. Segundo este teste obtém-se um limiar de rejeição, $X_{\rho, \kappa}^{2}$, onde $\rho$ representa o nível de 
significância do teste e $\kappa$ o número de graus de liberdade do problema. Para $X^{2}{ }_{0}$ $>X_{\rho, k}^{2}$, o modelo é rejeitado com nível $(1-\rho)$ de confiança, e a hipótese nula $\left(H_{0}\right)$ implica a aceitação do modelo log-normal para a volatilidade.

O teste de hipótese para $\alpha$ igual a dez por cento de probabilidade resultou em $H_{1}=0$, implicando na não rejeição da hipótese nula $H_{0}$.

Os resultados relacionados ao efeito de alavancagem estão de acordo com Gençay e Selcuk (2004), Bollerslev et al., (2006), e Cappa e Pereira (2010), que postulam que o efeito de alavancagem não é confirmado por dados de alta freqüência, indicando que em intervalos curtos de tempo não parece haver correlação bem definida entre volatilidade e movimentos de alta ou queda das ações de uma empresa.

Figura 6.- Melhor ajuste log-normal com a distribuição de volatilidade das ações CSNA3, calculado numa janela de tempo de 300 min.

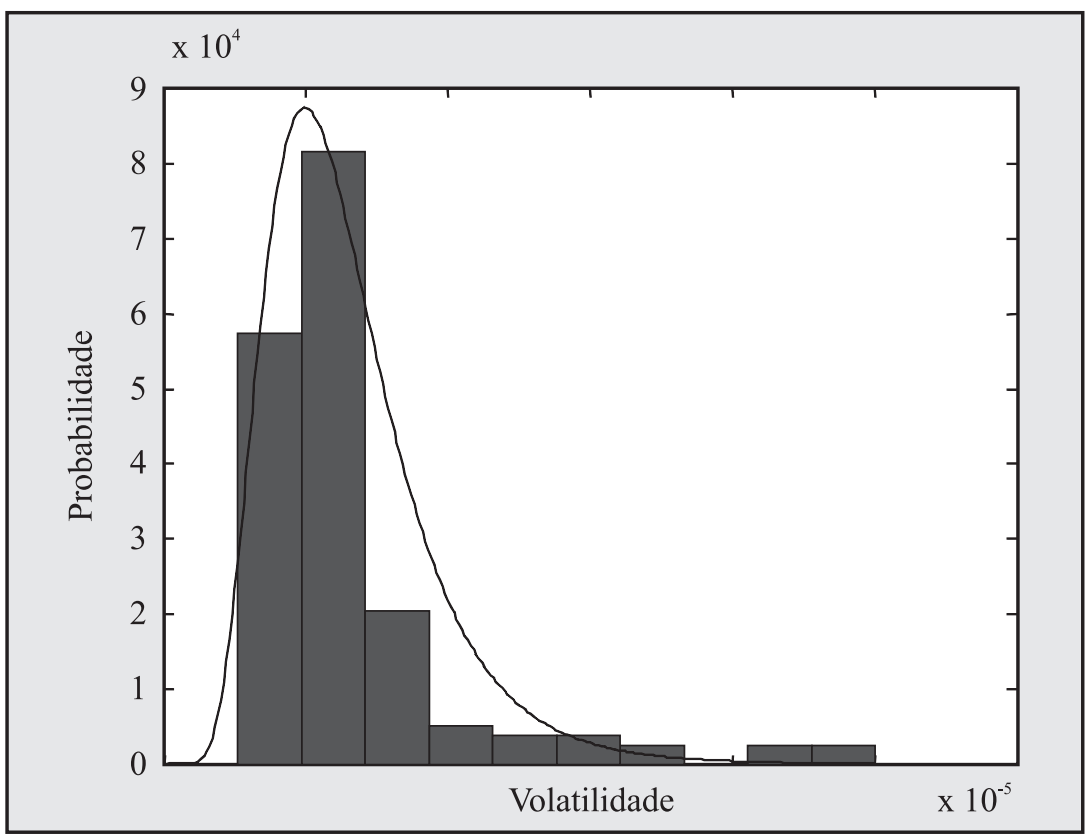

\section{Conclusão}

Neste estudo apresentou-se um conjunto de características estatísticas presente no mercado de ações brasileiro. Essas características emergem da análise empírica dos retornos de ações e da volatilidade das ações estudadas. 
As propriedades aqui mencionadas não resultam de uma hipótese paramétrica sobre o processo de geração de retornos, mas de hipóteses gerais de natureza qualitativa.

Juntamente com a apresentação dos histogramas dos retornos das empresas envolvidas no estudo, é mostrado o estudo da normalidade, realizado por meio do teste KS, no nível de significância $\alpha$ de cinco por cento de probabilidade. O referido teste apresentou, em todos os casos estudados, a não normalidade da distribuição.

As funções de densidade de probabilidade dos retornos dos diversos papéis analisados mostraram leptocurtose, com o grupo possuindo curtose média de 53,31 .

O estudo da assimetria permitiu verificar valores negativos para todas as empresas estudadas, implicando que a distribuição dos retornos é enviesada à esquerda.

Quanto à autocorrelação, o trabalho permite inferir a existência de ausência de autocorrelação dos retornos. Já a autocorrelação do módulo dos retornos apresentou um lento decaimento temporal, o que evidência a autocorrelação não linear dos retornos.

Para a comprovação da gaussianidade agregativa realizou-se o teste KS, no nível de significância de cinco por cento, sendo a hipótese nula $\left(H_{0}=0\right)$ a que confirma a normalidade dos dados. Obteve-se como resultado valor igual a 1 para os dados com amostragem de 20 minutos e 3 horas. Já para a amostragem de trinta e seis horas obteve-se zero como resultado. Ou seja, é possível concluir que existe gaussianidade agregativa para PETR4 quando o intervalo de tempo de coleta da amostra aumenta, no nível de significância de cinco por cento.

Quanto à volatilidade, a observação empírica mostra uma longa persistência da função de autocorrelação das volatilidades dos retornos. Após a eliminação dos agrupamentos de volatilidade, a série resultante ainda exibe caudas gordas, apesar de serem caudas menos pesadas que as apresentadas na distribuição original e de haver bom ajuste por meio de uma distribuição log-normal com a expectativa de convergência lenta para a distribuição gaussiana.

Para a correlação entre retorno e volatilidade, que caracteriza o efeito de alavancagem, não foram encontradas evidências que garantam a existência dessa característica.

\section{Referências}

BLACK, F. Studies of stock price volatility changes. Business and Economic Statistics Section. Proceedings from the American Statistical Association, Princeton, NJ, p. 177-181, 1976.

BM\&FBOVESPA. BM\&FBovespa: a nova bolsa. São Paulo: BM\&F, 2010. Disponível em: < http:// www.bmfbovespa.com.br/home.aspx?idioma=pt-br>. Acesso em: 08 set. 2012.

BOLLERSLEV, T.; LITVINOVA, J.; TAUCHEN, D. Journal of Financial Econometrics. Leverage and Volatility Feedback Effects in High-Frequency Data, v. 4, n. 3, p. 353-384, 2006. 
BOUCHAUD, J. P.; POTTERS, M. Theory of Financial Risk and Derivative Pricing From Statistical Physics to Risk Management, Cambridge: Cambridge University Press, 2003. 379p.

CAPPA, L.; PEREIRA, P. L. V. Modelando a volatilidade dos retornos de Petrobras usando dados de alta frequência. São Paulo: FGV-EESP, Jun. 2010 (Textos para discussão, n. 258)

CIZEAU, P. et al. Volatility distribution in the S\&P500 stock index, Physica A, v. 245, p. 441-445, 1997.

CONT, R. Empirical properties of asset returns: stylized facts and statistical issues. Quantitative Finance, v. 1, p.223-236, 2001.

FAMA, E. F. Efficient capital markets: a review of theory and empirical work. Journal of Finance, v. 25 , p 383-417, 1970.

GENÇAY, R.; SELCUK, F. Extreme value theory and Value-at-Risk: relative performance in emerging markets. International Journal of Forecasting, v. 20, n. 2, Apr./Jun. 2004, p. 287-303

GOPIKRISHNAN, P.; PLEROU, V.; AMARAL, L. A. N.; MEYER, M.; STANLEY, H. E. Scaling of the distribution of financial market indices, Physical Review E, v. 60, p. 5305-5316, 1999.

MANTEGNA, R. N.; STANLEY, H. E. An Introduction to Econophysics Correlation and Complexity in Finance, Cambridge: Cambridge University Press, 2000. 145p.

MORGAN, J. P. RiskMetrics - Technical Document. 4 ${ }^{\text {th }}$. Ed., New York, 1996.

PLEROU, V.; GOPIKRISHNAN, P.; AMARAL, L. A. N.; STANLEY, H. E.; GABAIX, X. Economic fluctuations and anomalous diffusion, Physical Review E, v. 62, n. 3, p. R3023-R3026, 2000.

SILVA, A. C.; YAKOVENKO, V. M. Stochastic volatility of financial markets as the fluctuating rate of trading: an empirical study. Physica A: Statistical Mechanics and its Applications, v. 382, n. 1, 2007, p. 278 - 285. Disponível em : URLhttp://www.sciencedirect.com/science//article/B6TVG NDMNBH3/2/2db88b37597ea91b42eefcb81a8c48a7. Acessado em 01 Fev. 2010.

SPIEGEL, M. R. Estatística. 2a . ed. São Paulo: McGraw-Hill, 1985. 454p. 